\title{
Pricing 50ETF Option Based on Genetic Algorithm BP Model
}

\author{
Yulin Du \\ Department of Finance, East China University of Political Science and Law, 201620, Shanghai
}

\begin{abstract}
Pricing financial derivatives is focus in finance theory and practice. Comparing to the traditional parameter model pricing method, the neural network method has obvious advantages in solving this problem. In this paper,we will price the option of Shanghai 50ETF based on the improved BP neural network model (GABP). The results show that the effect of neural network is better than that of B-S model, and the accuracy of GABP model is higher than that of BP neural network model and B-S model.
\end{abstract}

\section{Introduction}

Option is a kind of financial derivatives.Option holder has the right to buy or sell some assets (stocks, commodities and foreign exchange) at a certain time with a predetermined price. The option holder can exercise or give up this right in the validity period. For the option seller, it is obligated to perform the contract. Because the option gives the holder certain rights rather than obligations, so it has a certain value, and the option seller will be compensated for assuming obligations. Options include call options and put options, which give the holder the right to buy the underlying asset at a predetermined price at a certain time in the future, while the latter is given the interest to sell the underlying asset at a fixed price. At the same time, options can be divided into European options and American options according to the execution time. European options can only be exercised on the expiration date, while American options can be exercised at any time before the expiration date.

Since Black and Scholes put forward the first complete option pricing model (B-S model) in 1973, the theoretical and empirical research on option pricing has developed rapidly. B-S model is usually used to calculate the price of European options, and there are some restrictive assumptions, including: the underlying assets obey lognormal distribution; the risk-free interest rate is fixed during the period of option validity; the underlying assets are not paid dividends, and the yield variance is a fixed constant. However, the assumption of B-S model is too strict, which weakens the applicability of the original pricing formula in reality and makes it defective in theory and application. Therefore, all kinds of innovative option pricing models emerge as the times require and promote the development of option market. In order to avoid the defect of the traditional parameter option pricing model, and because the option pricing theory usually originates from the variables with nonlinear relationship, the artificial neural network method can be used to price the option. Artificial neural network (ANN) is a widely used non-parametric technology which need not make any assumptions about the distribution of the underlying assets. Through the artificial neural network model, wee can use the existing financial data to determine the structure and parameters of the model under the assumption of no parameter constraints.

\section{BP neural network of genetic algorithm}

\subsection{BP neural network}

BP (Back Propagation) network is a multilayer feed forward neural network, the network training, adjust the training algorithm right threshold follows the error backpropagation method. Usually the BP neural network has 3 layers or 3 layers these neurons, including input layer, hidden layer and output layer, realize the connection between the upper and lower layer, and the same neuron connection. The input layer neurons and neurons in the hidden layer and hidden layer between neurons and neurons in the output layer is the network weights, the connection strength between two neurons in the hidden layer. When a pair of learning is provided to the input neurons, neuronal activation values from the input layer through the hidden layer to the output layer, then according to reduce the error between the network output and the actual output samples from the output layer direction, reverse direction through a hidden layer to the input layer.

The structure of the BP neural network consists of the input layer, the hidden layer and the output layer, which is shown in Figure 1.

\footnotetext{
${ }^{\mathrm{a}}$ Corresponding author: tongjihz@sina.com
} 


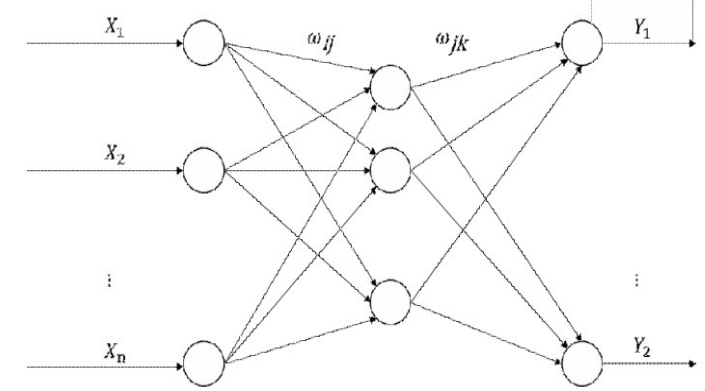

Input layer Hidden layer Output layer

Figure 1. The structure of BP neural network

$X_{1}, X_{2}, \mathrm{~L}, X_{n}$ is the BP neural network input value, $Y_{1}, Y_{2}, \mathrm{~L}, Y_{n}$ is to predict the value of the BP neural network, $\omega_{i j}, \omega_{\mathrm{jk}}$ are the weights of the neural network. BP neural network can be regarded as a nonlinear function, the network input and output respectively the function of the independent and dependent variables, as the network input nodes and output nodes are respectively $n, m$, which reflects a variable from the $n$ to $\mathrm{m}$ because of the mapping function variables in BP neural network.

\subsection{GABP neural network}

Genetic algorithm (GA) is a mathematical model designed to imitate the rules of natural selection. It was first proposed by Professor Holland in 1962. The model was then widely used in scientific research and engineering problems encountered in practice, especially in solving optimization problems. GABP process is divided into four parts: initialization, selection, crossover and mutation. The algorithm does not fall into local minimum when searching for the optimal value, and is a common method for global optimization. BP algorithm uses gradient method to search the optimal solution, which leads to its weak ability to search the optimal solution, easy to trap in the local optimal solution. The advantage of genetic algorithm is that it can quickly find the global optimal solution. The idea of combining the two is that binary coding is used to represent the weights randomly given by the network as the initial group, then the output value is calculated by using the weights of each group, then the fitness is calculated according to the output value, the probability of the network being selected is determined according to the fitness,so the process of genetic variation between the weights of the two groups is obtained. In this way, the new model (GABP) not only solves the problem of unstable output value of neural network, but also improves the training efficiency of the network.

The steps of using legacy algorithm to improve the neural network model are as follows:

(1)Generates a binary string set, which represents the set of connection weights. The relationship between binary weight and real weight is :

$$
\omega(i, j, k)=\omega_{\text {min }}(i, j, k)+\mathrm{b}(\mathrm{t})\left(\omega_{\max }(i, j, k)-\omega_{\min }(i, j, k)\right)
$$

Where $\mathrm{b}(\mathrm{t})$ represents the binary string, $\omega_{\max }(i, j, k)$ and $\omega_{\min }(i, j, k)$ represent the maximum and minimum of the join weight.

(2) An initial group of connection options is randomly generated and the binary connection weights are translated into real-valued weights.

(3) The output results can be obtained by combining the connection weights and input variables of real numbers. According to the size of the fitness to determine the probability of selecting the network, its probability value is:

$$
p=\frac{f_{i}}{\sum_{i} f_{i}}
$$

$P$ represents the network i-th in evolution of the selected probability, $f_{i}$ represents its fitness.

(4) According to the above probability value, two networks are randomly selected, and the two networks are crossed by a certain probability $\mathrm{P}$ (c is not zero) to produce a new network. Then the new network is mutated according to the probability of Pm (not zero), so the next generation network is obtained.

(5) repeat (2), (4) process until the required error can be reached.

\section{Empirical results and analysis}

\subsection{Data}

On February 9, 2015, Shanghai 50ETF option was listed on the Shanghai Stock Exchange, which is another kind of financial derivatives after the listing of Shanghai and Shenzhen 300 stock index futures. Since the listing of Shanghai 50ETF options has been developing rapidly, investors have been paying more and more attention to it. Statistics from the Shanghai Stock Exchange show that the monthly turnover of the 50ETF options has gradually increased, from about 230,000 contracts in February 2015 to 23.86 million contracts in December 2017, an increase of more than 100 times in three years. Among them, the subscription options contracts have increased 108 times and the put options contracts have increased 97 times. The number of open options contracts on the Shanghai Stock Exchange 50ETF also increased rapidly, from 40,000 in February 2015 to 1.47 million in December 2017 , an increase of about 36 times, with subscription and put contracts increasing by 38 times and 33 times respectively. For the continuity of the data, we select the first 20 days of the main contract 50ETF purchasing August 2600 as the training data, and the next 15 days as the test set to test the prediction effect of models, datas are from the WIND database, below is the graph of its price. 


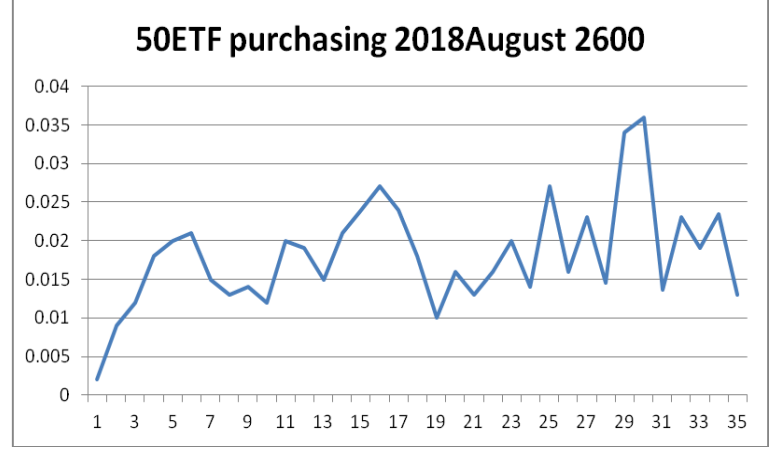

Figure 2. Price of 50ETF purchasing 2018August 2600

\subsection{Model empirical results and accuracy comparison}

We simulate the price of 50ETF purchasing August 2600 with Black-Scholes formula, BP neural network and GA BP neural network. The results are as follows:

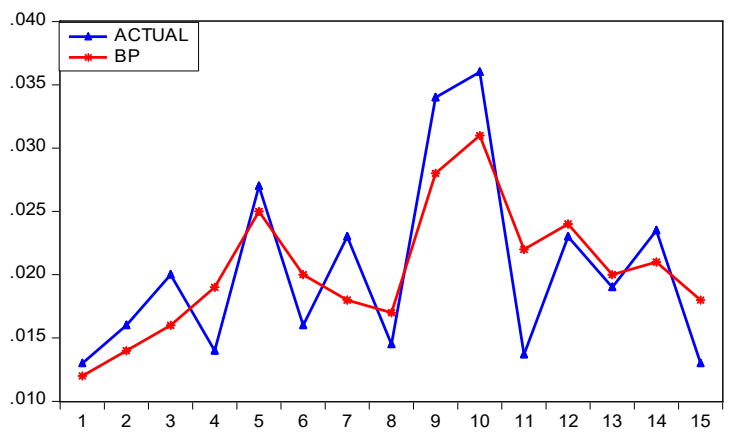

Figure 3. Result of BP Neural Network model

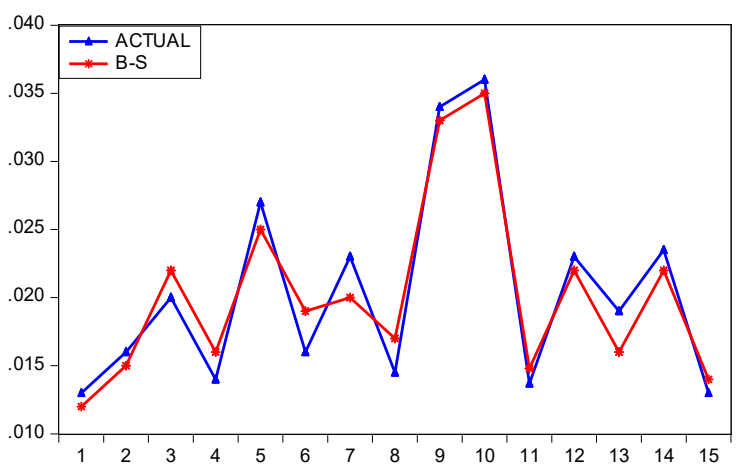

Figure 4. Result of Black-Scholes model

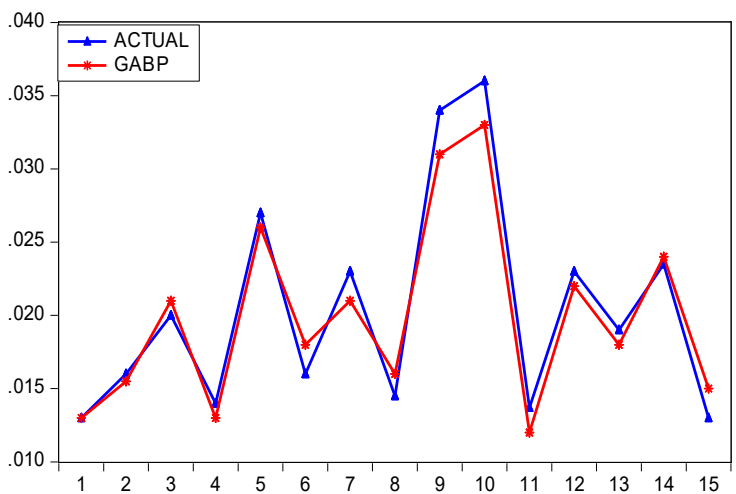

Figure 5. Result of GABP model
In order to verify the predictive ability of the three models, we measure the predictive effect of the three models by means of the root mean square error and the relative mean error. The mean square error is the expected value of the square of the difference between the parameter estimation and the true value of the parameter ( Root Mean Squared Error, RMSE).

$$
R M S E=\sqrt{\frac{1}{n} \sum_{i}\left(\hat{y}_{i}-y_{i}\right)^{2}}
$$

The mean relative error is better to reflect the actual situation of the prediction error because it has no dimensional influence, it is a percentage easy to make comparison

$$
M R E=\sqrt{\frac{1}{n} \sum_{i}\left(\frac{\hat{y}_{i}-y_{i}}{y_{i}}\right)^{2}}
$$

Table 1. MRE and RMSE of three models

\begin{tabular}{|c|c|c|}
\hline Methods & $M R E$ & $R M S E$ \\
\hline BP & $10.31 \%$ & 0.0033 \\
\hline Black-Scholes & $7.91 \%$ & 0.0018 \\
\hline GABP & $5.18 \%$ & 0.0009 \\
\hline
\end{tabular}

From the result of the model, RMSE and MRE results of GABP is the smallest among the three kinds of models, the results of the BS model is in the middle, the worst is the results of BP, so GABP's rediction accuracy is much higher than that of $\mathrm{BS}$ and $\mathrm{BP}$ neural network, the change of option has obvious non-linear characteristics.

\section{Conclusions}

Through the empirical process, we find that the results of genetic $\mathrm{Bp}$ algorithm is the best, its simulation and prediction accuracy is higher than BS model and BP neural network model, so the model has certain advantages in prediction.

\section{References}

1. Chang-Sheng L I, Hai S, Li X, et al. Prediction method of formation fracture pressure based on BP neural network optimized by genetic algorithm( GA)[J]. Journal of Xian Shiyou University, 2015

2. Huang Z, Zheng G, Jia Y. Forecasting Exchange Rate Volatility with Linear MA Model and Nonlinear GABP Neural Network[C]// International Conference on Business Intelligence \& Financial Engineering. IEEE, 2012:22-26.

3. Jiang L. The Applications of GA-BP Neural Networks in Option Direction for Catalytic Agent[J]. Applied Mechanics \& Materials, 2014, 510:45-50.

4. Li W, Yan T, Liang $Y$ J. Pressure Prediction Technology of the Deep Strata Based on BP 
Neural Network[]]. Advanced Materials Research, 2010, 143-144(2):28-31.

5. Mitra S K. An Option Pricing Model That Combines Neural Network Approach and Black Scholes Formula[J]. Global Journal of Computer Science \& Technology, 2012.
6. Samur, Zeynep İltüzer, Temur, Gül Tekin. The Use of Artificial Neural Network in Option Pricing: The Case of S\&P 100 Index Options[J]. World Academy of Science Engineering \& Technology, 2009(54):326. 